\title{
Barriers and Facilitators to Modern Contraception Among Married Women in Conflict Affected Town of Mogadishu, Somalia
}

Abdi Gele ( $\sim$ abdi.gele@fhi.no )

Norwegian Institute of Public Health

Mary Shrestha

Save the Children

Fathia Khalif

Church City Mission

Samera Qureshi

Norwegian Institute of Public Health

\section{Research Article}

Keywords: Reproductive health utilization, family planning, Sub-Saharan Africa, conflict setting

Posted Date: May 27th, 2021

DOI: https://doi.org/10.21203/rs.3.rs-551043/v1

License: (c) (i) This work is licensed under a Creative Commons Attribution 4.0 International License. Read Full License 


\section{Abstract}

Background - With a maternal mortality ratio of 692 per 100,000 live births and modern contraception prevalence of $1 \%$, understanding factors hindering Somali women from using modern contraception is key to developing and implementing locally adopted public health responses. The purpose of this qualitative study is to explore barriers and facilitators to the use of modern contraception among married women in Mogadishu.

Methods - We conducted a qualitative study using semi-structured, in-depth interviews with 21 married women aged >18 years, living in different neighborhoods in Mogadishu between July-December 2018. We recruited the participants using a convenience sampling method. We used thematic analysis and adapted ecological model as a framework for the synthesis.

Results - The findings show that systemic, individual and socio-cultural barriers hinder women's ability to access modern contraception. Barriers identified by this study include: Health communication messages and contraceptive information provided by health providers, prevalent religious fallacies among women and fear of permanent infertility upon modern contraception.

Conclusion - Training health providers in the principles of modern contraception, in addition to the medical ethics that govern their responsibility to provide correct and relevant information to their patients, is vital for increasing modern contraception use among Somali women. The findings of this study may be used for designing public health interventions that promote acceptance and the use of modern contraception among both women and men in Somalia.

\section{Introduction}

It is widely reported that women living in countries affected by conflicts predominantly experience unintended or unwanted pregnancies due to a lack of access to contraceptive services. The ten countries with the highest maternal mortality ratios in the world are countries affected by war and they are characterized by low contraceptive prevalence (1). Despite the high demand for birth spacing or limiting the childbirth in conflict-affected populations (2), there is limited contraceptive information and services available for women and girls living in these contexts (3). According to the World Health Organization (WHO), tailored contraceptive services are not only critical to improving the health of women, but it is also a human right. There are humanitarian organizations such as UNFPA that provide long-acting and permanent contraception methods but most available services in humanitarian settings are limited to short-acting methods $(4,5)$. Therefore, many women who want to postpone or not have a pregnancy again are unable to obtain the desired contraceptive methods in conflict settings.

Somalia is an East-African country with a population of 12 million, with women living in Somalia having among the worst maternal and reproductive health outcomes in the world (4). According to the Somali Demographic Health Survey (SDHS), only $7 \%$ of currently married women were using any contraceptive method, with only $1 \%$ using modern contraception (4). The contraceptive methods that are available in 
Somalia include injectables and oral pills. Long-acting reversible contraceptives (LARC) are also available in few health facilities run by NGOs and few hospitals. Despite the availability of LARC, a study on postabortion use of family planning found that Somali women prefer short-term methods because of the desire of most women to return to fertility as soon as possible after abortion (6). In Somalia, large families are valued and, therefore, women often experience peer pressure to bear more children (7). Further, knowledge and understanding of modern contraception is low amongst Somali women, including those who wish to avert pregnancy (8). As a result, Somali women's fertility rate is 6.9 (4), exceeding both the fertility rate in Sub-Saharan Africa (5.1) and the world (2.5). The high fertility reflects the high maternal mortality ratio of Somalia (692 per 100,000), which is much higher than the average in SubSaharan Africa $(84.5$ per 100,000) (9). The potential impact of contraceptive use on maternal deaths in Somalia is unknown, although global estimates show that $35 \%$ of maternal deaths could be averted by preventing unintended pregnancies (10). To our knowledge, few studies have investigated the issue of contraception in Somalia $(6-8,11)$, but none of these studies have investigated barriers to contraception use among married women. In Somalia, where prevalent negative attitudes toward abortion, as dictated by religion and culture, hamper access to safe abortions; more rigorous and effective contraceptive programs should be the current approach to help reduce the risk of unwanted pregnancies and unsafe abortions. The aim of this study is to explore barriers and facilitators to contraception use among married women in Mogadishu, Somalia, and to inform national reproductive health programs.

\section{Methods}

\subsection{Sample and Sampling technique}

We conducted a qualitative study using semi-structured in-depth interviews in Mogadishu from July to December 2018. Mogadishu is the capital of Somalia, with a population of two million. Most of the health services in the country are concentrated in Mogadishu. We therefore chose semi-structured interviews, because it is not only a flexible tool for exploring people's experiences and their attitudes of reality, but it is also a tool that does not impose a priori categorization of the questions, which may thus limit the field of inquiry (12). We recruited 21 Somali women, all married, aged $>18$ years, from different settings using a convenience sampling technique. Three local researchers and FKM participated in the recruitment. They recruited information rich cases from their acquaintances, family, friends and networks through telephone contact first, and then met at their homes. The Norwegian Regional Committee for Medical and Health Research Ethics, and the ethical committee at the Somali National University, approved the study with approval number: $2017 / 2386$. Because many of the participants were illiterate, we obtained oral consent from all the participants.

\subsection{Data collection}

We applied an interview guide previously used among Somali women in Oslo (13)], pre-tested in Mogadishu, and iteratively adapted as the study progressed, to 21 married women living in Mogadishu. We interviewed participants individually in the Somali language at their preferred choice of place, mostly 
at their homes. As shown in Table 1, we asked the participants questions about their demographic characteristics. Due to the semi-structured interview technique with open-ended questions, women could go beyond the actual questions and talk freely about their experience regarding contraception methods, and to highlight additional issues of concern, which allowed discussion of previously unplanned themes. We used the term, "daawooyinka ilmaha lagu kala dheereeyo," which means child spacing treatment/drugs and "uurka la isaga ilaaliyo," which means treatment/drugs for the prevention of pregnancy. The study therefore focused on modern contraception (hereafter referred to as "contraception"). The interview guide covered two themes: (1) reasons for usage or non-usage of contraception; and (2) factors that prevent Somali women from utilizing of contraception. The interviews lasted between 45 to 60 minutes, and we audio-recorded all the interviews after obtaining the participants' consent. The author FKM, who is a female research assistant with extensive experience in qualitative data collection, carried out the interviews. We continued the recruitment and interviews until the researchers were confident that saturation was achieved with 21 cases (14). 
Table 1

Demographic characteristics of the married female study participants

\begin{tabular}{|c|c|c|c|c|}
\hline S.N. & Age & Employment & Education & Number of children \\
\hline 1 & 26 & Business & No education & 5 \\
\hline 2 & 39 & Unemployed & Secondary & 8 \\
\hline 3 & 24 & Unemployed & No education & 3 \\
\hline 4 & 27 & Unemployed & No education & 5 \\
\hline 5 & 39 & Unemployed & No education & 12 \\
\hline 6 & 40 & Unemployed & No education & 11 \\
\hline 7 & 22 & Student & Secondary & 2 \\
\hline 8 & 31 & Unemployed & Primary & 7 \\
\hline 9 & 28 & Unemployed & Primary & 6 \\
\hline 10 & 22 & Unemployed & Primary & 3 \\
\hline 11 & 19 & Unemployed & Primary & 2 \\
\hline 12 & 23 & Unemployed & No education & 4 \\
\hline 13 & 19 & Unemployed & Primary & 3 \\
\hline 14 & 37 & Unemployed & Secondary & 9 \\
\hline 15 & 29 & Unemployed & No education & 6 \\
\hline 16 & 36 & Employed & Secondary & 8 \\
\hline 17 & 30 & Unemployed & No education & 5 \\
\hline 18 & 37 & Business & No education & 10 \\
\hline 19 & 21 & Student & College & 2 \\
\hline 20 & 26 & Unemployed & No education & 5 \\
\hline 21 & 39 & Unemployed & No education & 7 \\
\hline
\end{tabular}

\subsection{Analysis}

FKM transcribed the interviews verbatim. During the data collection and analysis, two female medical students based in Mogadishu, together with a male researcher, assisted the research team with participant recruitment, interview design and revision, as well as verification of the accuracy and validity of emerging themes. We translated the transcripts into English for coding. Coding was done in two rounds. First, the authors, AG and FKM, carefully read the transcripts several times for accuracy and completeness. The first and second authors (AG and MS) then reviewed the coded excerpts and 
quotations to help understand the link between different concepts, and eventually develop core categories. We later used the constant comparison method and selective coding to identify emergent themes within and across interviews. Our purpose was to identify and confirm the most prominent themes, search for any inconsistencies, develop connections between the present study and other similar studies in the field, and to reflect on persistent ambiguous or contradictory issues requiring careful consideration. We used thematic analysis to identify and analyze important categories and themes (15). Lastly, we divided the themes that emerged from the women's stories into categories based on the participants' experiences of factors impeding or facilitating their access to contraceptive use. All of the authors reviewed, commented and provided input on the final themes.

Finally, we used the modified ecological model for the thematic analysis as this model helps understand and interpret various factors that influence the health behaviour and helps better organization of the themes (16). AG and MS examined the final agreed themes in relation to the ecological model domains and considered how each theme contributed to our understanding of levels of this model. In this analysis, the focus was on individual, socio-cultural and health system related barriers and a facilitator to uptake of contraception among Somali women. All of the authors reviewed, commented and provided input on the final themes.

\section{Results}

We identified factors that may impede women's access to modern contraception. These factors were compiled into four major themes (Fig. 1): Health system related barriers, individual barriers, socio-cultural barriers and a facilitator. Many women who wanted to access modern methods in health care found that health system-related and individual barriers hindered their access and acceptance to contraception utilization. Furthermore, women reported how religious and cultural factors impacted on their ability to access to contraceptive methods. Nevertheless, most women expressed that contraception is helpful for child spacing, which is the only facilitator reported by women.

\subsection{Health system related barriers}

\subsubsection{Health provider's demand for husband's approval and presence}

All of the participants in the study mentioned a prevalent system in the health services, whereby health providers require the husband's presence and approval to provide injections or any other contraception to women, with the exception of Oral Contraceptive Pills (OCP). This mandatory requirement as part of the health system creates a limitation in choosing multiple options of contraceptive services,, even when the services are available free of charge. All women reported that health providers require the husband's approval, due to the fear that if the husband becomes aware that his wife has received contraception, and she is accordingly unable to conceive, he may violently confront the doctor who provided the contraception. One participant reported that in cases in which the doctor is willing to provide modern 
contraception, the doctor often takes an oath with the woman that if her husband finds out that she used contraception, she must not reveal the identity of the doctor who conducted the procedure.

"When they gave the injection, their last word is always like; look! 'We did not see each other', ok? Therefore, women jokingly call the injection (Isma arkin), 'We did not see each other.' I think the doctor is scared of the husband and relatives. They always say that if your husband gets to know that you have got an injection and come to me, I will deny and tell him that I have never seen this woman." (Participant 1)

"Your husband must be with you when receiving the injection, he must approve it, and give permission. However, the pill is available without condition." (Participant 5)

One of the participants reported the availability of free contraception at certain mother and child clinics, but that the clinics' requirement for the husband's presence and approval may make women afraid to use their services.

"There are few Mother and Child Health Clinics that provide free contraception, but the provision of the method requires the husband's presence and approval." (Participant 2)

\subsubsection{Doctors discourage contraceptive usage instead promote breastfeeding}

The participants reported that doctors discourage them from using contraception because of side-effects that doctors perceive to be associated with these methods. Accordingly, several participants highlighted that they are afraid of-, and have concerns about contraception.

"I and my husband have discussed once about contraception. I had anemia. We met a doctor, and the doctor told us that it causes bleeding and health problems, so I should not use it. It is Dr. XX who is well known in our area." (Participant 11)

"I sought contraception once, and the doctor scared me about using it, and I decided not to use it." (Participant 21)

Most of the study participants reported hearing once or on several occasions from doctors expressing that the prolonged use of contraception was the reason that some Somali women became infertile. In a context in which children are highly valued, and are regarded as providing welfare for the family, this type of information is enough to discourage women from using contraception regardless of its availability.

"Doctors told that some women may never become pregnant because they used contraception for a long period." (Participant 19)

Many women reported that doctors have advised them to use traditional child spacing methods, particularly Lactation Ammorhoea Method (LAM), as the only acceptable and safe method for delaying or 
stopping pregnancy. Accordingly, many participants demonstrated confidence in LAM. This has thus, made the women reluctant in using contraceptive services as per the advice of the doctors.

"The doctor advised me to use breastfeeding for child spacing, that is the only method the doctor told me." (Participant 12)

\subsubsection{Provider's requirement for a medical condition that justifies the provision of contraception}

We asked women if they currently use contraception, and most of them responded that they had no medical conditions that made it necessary to seek contraceptive methods. Almost all women reported that the use of contraception could only be justified when medical conditions such as cesarean delivery require the delay of the next pregnancy. According to the participants, having closely spaced children was not enough to justify the use of contraception, unless it was accompanied by observable complications.

"If a woman has had a caesarian section, the doctor prescribes contraception for her, but if someone has not experienced a cesarean delivery before then, the doctor will not prescribe contraception." (Participant 18)

"My children are spaced by one year to one and half years. I did not experience any problems in delivery. People who experienced cesarean delivery or other health problems need to use contraception. Doctors never advised me to use contraception because I have not had any problem." (Participant 8)

\subsection{Socio-cultural barriers}

\subsubsection{Contraception is perceived as a foreign culture}

The study participants considered contraception to be an imported Western tradition, which is against their norms and traditions, thereby hindering its usage. The participants blamed foreigners for being the perpetrators of introducing this culture into Somalia.

"I have not seen anybody supporting modern contraception use. Some contemporary women talk about it, but it has nothing to do with our culture. Women used to space children traditionally, using contraception is a foreign culture." (Participant 14)

"Some people use contraception because they follow the Western tradition, but most Somalis do not use contraception." (Participant 3)

\subsubsection{Contraception is perceived as being non-Islamic}

In Mogadishu, where people see contraception as non-Islamic and foreign, it is not inexplicable for women to hold negative religious beliefs and opinions against contraception. The use of contraception is seen as a platform contrary to God's plan. There are beliefs that it does not matter how many children one has; God cares and provides for all the children who are born. 
"I do not see it as a good thing. For me, it is good to rely on God's plan. God can stop you from conceiving if he wants. Therefore, it is wrong to use contraception." (Participant 11)

Other participants thought that contraception was for non-believers, and that the use of contraception might draw God's anger, which could result in collective punishment to their community. This type of fear prompted women to remain defiant about using contraception. Women reported that the use of contraception meant challenging God's plan, which might result in undesired negative effects, such as permanent infertility or unknown illnesses.

"Those illnesses that are widespread today did not exist before these drugs came into our country. People can space their children without using contraception." (Participant 4)

"I want to have many children. People are telling that they use contraception, which is arrogance against God's natural process. They may never have children again." (Participant 13)

\subsection{Individual barriers}

\subsubsection{Fear of permanent infertility upon usage of modern contraceptive methods}

Most participants mentioned the potential reason for not favoring contraception is the association between contraception and irreversible infertility, with subsequent risk for family breakdown.

My cousin lived in the USA, she had two kids. Her husband suggested her to use injection. She never had a child again. Her husband came to Somalia and married another woman. (participant 4)

It is possible that if you use contraception you may experience health problems. I saw people who used contraceptives and who, consequently had bleedings. Others have stopped having menstruation. It is also possible that it affects the eggs and sometimes you may not become pregnant. (participant 19)

I do not use it because I have heard people talking about that they may not have any children after using it. (participant 12)

\subsection{Facilitators to the use of contraception}

\subsubsection{Contraception is important for child spacing}

Despite the existing tradition of having many children, the participants had a positive attitude and very good understanding toward the importance of child spacing. They expressed that contraception allowed an adequate amount of time for a child to be breastfed. Nevertheless, even women who had given birth to as many as 12 children had the opinion that their children were correctly spaced. Almost all of the participants expressed the desire to have as many children as they could, but also understood the benefits of child spacing. 
"Children require being breastfed for two years, so those drugs (contraception) for child spacing are important for women who cannot space their children through breastfeeding (LAM). Women need to have time to relax from the effect of the previous pregnancy, and contraception may give them that opportunity." (Participant 15)

"...my children are nicely spaced (12 children). As long as the child is breast feeding, I do not become pregnant... so those treatments for child spacing are important for women who cannot space their children through breastfeeding." (Participant 5)

\section{Discussion}

The study has explored barriers and facilitators to the use of modern contraception in Mogadishu, Somalia. Although research has documented that pregnancies occurring within a year of the mother's previous birth are riskier for the health of both the mother and child (17), only $7 \%$ of women in Somalia use any contraception, and only $1 \%$ use modern methods. Our findings show that health providers' request for husbands' presence and approval, has severely affected women's access and usage of modern methods. The doctors' behavior may be dictated by the prevalent social norms, exacerbated by their limited knowledge on reproductive health (11), which may make them believe that without their husbands' approval, contraception cannot be prescribed to women. However, this behavior violates women's right to privacy, and to making autonomous decisions pertaining to their health and fertility. According to the United Nations Population Fund (UNFPA), women's right to contraceptive information and services is grounded in internationally recognized human rights, including the right to decide the number and spacing of one's children, the right to privacy, the right to information and the right to equality (18). Moreover, it was quite surprising that doctors discourage women from using modern contraception, instead encouraging the use of traditional methods such as LAM. While we must not underestimate the importance of LAM for family planning, the resistance of the use of modern methods among women could be due to health providers' inaccurate beliefs about contraception. A previous study conducted in Mogadishu has highlighted health professionals' misunderstanding about contraception, which was attributed to the education system in which health professionals were trained (11). Further, challenges in family planning implementation in Somalia include low knowledge and skills of service providers especially in modern contraceptives in general (19).

The present study demonstrates that there is a popular norm that contraception can only be sought if a woman has undergone a cesarean delivery. Somali culture is in favor of a family having many children. Medical doctors seem to be supporting and enforcing that culture by discouraging women from preventing pregnancy unless there is a health problem. A study reported that providers who were unaware of the WHO recommendation to administer contraception were more likely to inappropriately limit its use in their patients (20). In agreement with previous studies (11), it is vitally important to train health providers in regard to the importance of contraception for women and children's health, as well as the obligation for medical professionals to provide correct and relevant information to women under their care. 
In line with a prior study (21), the present study reports individual barriers such as misunderstandings resulting in a fear of the side-effects of modern contraception, which is another hindrance for the uptake of the contraception. The primary side-effect that the participants associated with contraception was infertility; they have received this information from health providers, while it is also a prevalent perception in Mogadishu. In Somalia, where the reason for marriage is mainly for having children, anything that is suspected of jeopardizing that goal is completely avoided. Health providers are part of society, and may have succumbed to what the majority of the people believe instead of using their medical judgement and knowledge regarding the benefit of contraception. The individual barriers to contraception in this study is further affected with the information provided at the health system level. Women's barriers to contraception that is based on unfounded information given by health providers are called medical barriers (22). A previous systematic review documented that contraceptive use, regardless of its duration and type, does not have a negative effect on the ability of women to conceive following termination of use, and does not significantly delay fertility (23). Because contraception is safe depending on the individual situation, appropriate counseling is important to ensure women use modern contraception suitable to their situation and requirements.

The socio-cultural barriers where women perceive that modern contraception is a non-Muslim practice is another hurdle to contraception use among women in Somalia. In line with a study in Tanzania, the use of family planning was perceived as against the teaching of faith and commands (21). Study participants believed that they should have as many children as God planned for them. While all four Sunni schools of thought agree on family planning, a prior review categorized current religious interpretations regarding family planning into two different subjective thoughts: a group that openly accepts and promotes the use of modern contraception $(24,25)$ as unanimously instructed by the four Sunni schools, and a group that strongly opposes them, except when used for medical reasons (26-28). Accordingly, and in line with our study, prior studies report that the use of contraception is believed by women to be acceptable in Islam when it is used for child spacing, though not for fertility control (2628). Addressing religious barriers regarding contraception use is critical in reproductive health programs, and should be addressed using tailored approaches in collaboration with religious leaders.

In contrast, participants demonstrated only one facilitator for the uptake of contraception. Women showed a high level of understanding about the importance of child spacing to improve the health of both the mother and child. This finding is in accordance with prior findings that Somali women believe that Islam supports the practice of child spacing for health reasons. For this purpose, from a religious perspective, Somali women preferred natural contraception methods such as LAM and the withdrawal method (29). Despite this understanding, the participants had an average of six children, thereby believing that their children were properly spaced. According to the SDHS, the average fertility rate in Somalia is 6.9 children per woman, with $91 \%$ of Somali women considering six or more children to be the ideal family size (4). This may be explained by social norms around childbearing in Somalia and the entrenched patriarchal privileges that place decision-making powers in men's hands, with women having to comply with men's demand for many children (21). To avoid adverse outcomes associated with closely spaced births, the uptake of contraception was recommended (30). However, the participants' positive 
attitude towards child spacing has failed to translate into decisions to use contraception. This finding is in agreement with prior findings that the use of contraception is not just a matter of knowledge or rational choice by a person, but it is often mediated by social norms and power relations based on gender and ethnicity (31).

This study has some limitations. The purposive selection of the study sample does not ensure that these populations were representative of all Somali women in Mogadishu. Moreover, study participants were women who predominantly had a low education and were unemployed. Therefore, educated women may experience different barriers not mentioned here. Other studies have investigated barriers to modern contraception among Somali communities, both in Somalia (32) and among Somali immigrants in the West, and found similar results $(13,29)$. Our study focused on modern contraception and left traditional methods unaddressed. This may devalue the traditional methods such as LAM, which are effective, and which many Somali women use for child spacing. The study findings are in line with our previous quantitative findings, thus ensuring the trustworthiness of the findings (7). Contextual, linguistic and cultural knowledge of data collectors and researchers may also contribute to the reliability of the data (33). Furthermore, transcripts were made by the FKM, with all the other authors agreeing on codes and themes that ensure the confirmability of the data. The aim of the study is not to generalize the findings in any way, which makes this study non-transferable. Despite these limitations, the result of this study contributes to the existing body of knowledge on access to modern contraception among women in a humanitarian setting. The findings of this study can be used for designing a tailored intervention aimed at health providers, women and men, to improve the utilization of modern contraception in Somalia.

The implication of this finding is that the narratives provided by participants may help reproductive health providers, government institutions and civil society organizations to better understand the systemic, cultural and social contexts affecting Somali women's modern contraceptive decisions and behaviors. Health communication messages and contraceptive information provided by health providers are key factors influencing the health behavior of Somali women. Therefore, training health providers in the importance of modern contraception and medical ethics, which govern their responsibility to provide correct and relevant information to their patients, is vital for increasing access to contraception among Somali women. Families should receive accurate information and counselling services regarding modern contraceptive methods. Moreover, knowledge about the risks associated with closely spaced children and pregnancy-related risks should be improved in both women and health providers, given the fact that the risk perception of both women and health providers dictates subsequent decision-making regarding contraceptive use. Future research should focus on public health interventions that promote culturally tailored, modern contraception health promotion for women and men in Somalia.

\section{Declarations}

\section{Ethical approval}


Ethical approval for the study was obtained from Norwegian Regional Committee for Medical and Health Research Ethics and the ethical committee at Somali National University with approval number:

$2017 / 2386$. Participants were informed about the aims of the study, the confidentiality of their responses, and their rights to withdraw from the study at any time and without giving any reason. All participants in this study were $>18$ years. Therefore, informed consent to participate in the study was obtained from all participants prior to their participation. All methods were performed in accordance with the relevant guidelines and regulations.

\section{Consent for publication}

Authors consent the publication of the paper

\section{Availability of data material}

The datasets generated and/or analyzed during the current study are not publicly available due to ethical guidelines that we signed prior to data collection but are available from the corresponding author on reasonable request.

\section{Competing interests}

The authors have declared that no conflicts of interest exist.

\section{Funding Statement}

This work was supported by Dam stiftelsen

\section{Authors' Contributions}

AG. Secured the funding, analyzed data, and wrote the manuscript

MS. Analyzed the data, prepared tables and figures and participated in article write-up

FM. Collected the data, participated in data analyses, and commented the paper

SQ. participated in the write up of the article and analysis

\section{Acknowledgements}


The authors acknowledge the help they received from DAM stiftelsen. We also thank Vera Minja for helping the research team with administrative work, and Abdi Salad, Moallim Sa'diyo, Mohamed Saynab and Mohamud Farhiya for helping with the recruitment and collecting the data.

\section{References}

1. WHO, UNICEF, UNFPA, World Bank. Trends in maternal mortality: 1990-2013. Estimates by WHO, UNICEF, UNFPA, The world bank and the UN population division. Geneva: WHO; 2014. http://apps.who.int/iris/bitstream/10665/112682/2/9789241507226_eng.pdf, .

2. McGinn T, Austin J, Anfinson K, Amsalu R, Casey SE, Fadulalmula SI, Langston A, Lee-Jones L, Meyers J, Mubiru FK, et al. Family planning in conflict: results of cross-sectional baseline surveys in three African countries. Confl Heal. 2011;5:11.

3. Casey SE, Chynoweth SK, Cornier N, Gallagher MC, Wheeler EE. Progress and gaps in reproductive health services in three humanitarian settings: mixed-methods case studies. Confl Heal. 2015;9:S3.

4. Federal Government of Somalia/UNFPA Somalia. Somalia Demographic Health Survey. 2020. https://somalia.unfpa.org/en/publications/somali-health-and-demographic-survey-2020.

5. Huber D, Saeedi N, Samadi AK. Achieving success with family planning in rural Afghanistan. Bull World Health Organ. 2010;88:227-31.

6. Chukwumalu K, Gallagher MC, Baunach S, Cannon A. Uptake of postabortion care services and acceptance of postabortion contraception in Puntland, Somalia. Reproductive health matters. 2017;25(51):48-57.

7. Gele AA, Musse FK, Qureshi S. Unmet needs for contraception: A comparative study among Somali immigrant women in Oslo and their original population in Mogadishu, Somalia. PLoS ONE. 2019; 14(8): e0220783. https://doi.org/10.1371/journal.pone.0220783.

8. Gure F, Dahir MK, Yusuf M, Foster AM. Emergency Contraception in Post-Conflict Somalia: An Assessment of Awareness and Perceptions of Need. Studies in family planning. 2016;47(1):69-81.

9. Bongaarts J, Bruce J. The causes of unmet need for contraception and the social content of services. Studies in family planning. 1995;26(2):57-75.

10. Population growth and the Millennium Development Goals. Potts M, Fotso JC. Lancet. 2007 Feb 3; 369(9559):354-5.

11. Yalahow A, Hassan M, Foster AM. Training reproductive health professionals in a post-conflict environment: exploring medical, nursing, and midwifery education in Mogadishu, Somalia. Reprod Health Matters. 2017 Nov;25(51):114-123. doi: 10.1080/09688080.2017.1405676. Epub 2017 Dec 6. PMID: 29210333.

12. Svend Brinkmann \& Steinar Kvale. InterViews: Learning the Craft of Qualitative Research Interviewing. Third adition, 2014. SAGE Publications Inc. Thousand Oaks, United States.

13. Gele A, Fathia K Musse and Mary Shrestha. Barriers and facilitators to contraceptive usage among Somali immigrant women in Oslo: A qualitative study. Plos One (2020) 
14. Jill J. Francis et al. What is an adequate sample size? Operationalising data saturation for theorybased interview studies. Psychology \& Health Volume 25, 2010 - Issue 102010.

15. Braun V, Clarke V. Using thematic analysis in psychology. Qualitative Research in Psychology. 2006;3(2):77-101. .

16. McLeroy KR, Bibeau D, Steckler A, Glanz K. An ecological per-spective on health promotion programs. Health Educ Q. 1988;15(4):351-377.

17. DaVanzo $\mathrm{J}$ et al., Effects of interpregnancy interval and outcome of the preceding pregnancy on pregnancy outcomes in Matlab, Bangladesh, British Journal of Obstetrics and Gynaecology, 2007, 114(9):1079-1087.

18. Center for Reproductive Rights, United Nations Population Fund. The right to contraceptive information and services for women and adolescents 2010. Available; https://www.unfpa.org/sites/default/files/resource-pdf/Contraception.pdf.

19. Ali Sheikh Omar. Country Worksheet: Prioritized Action Planning 2019-2020 Somalia /Somaliland. Available: https://www.familyplanning2020.org/sites/default/files/SomaliaSomaliland_Worksheet.pdf.

20. World Health Organization. Medical Eligibility Criteria for Contraceptive Use. 4th edition. Geneva, Switzerland: WHO; 2004.

21. Agnes Cyril Msoka, Eunice Siaity Pallangyo, Sharon Brownie, Eleanor Holroyd. My husband will love me more if I give birth to more children: Rural women's perceptions and beliefs on family planning services utilization in a low resource setting. International Journal of Africa Nursing Sciences. Volume 10, 2019, Pages 152-158.

22. Bertrand JT, Hardee K, Magnani RJ, Angle MA. Access, quality of care and medical barriers in family planning programs. Int Fam Plan Perspect. 1995;21:64-74.

23. Tadele Girum, Abebaw Wasie. Return of fertility after discontinuation of contraception: a systematic review and meta-analysis. Contracept Reprod Med. 2018; 3: 9.

24. Haider S, Todd C, Ahmadzai M, Rahimi S, Azfar P, Morris J, et al. Childbearing and contraceptive decision making among afghan men and women: a qualitative analysis. Contraception. 2008;78(2):184.

25. Hughes CL. The "amazing" fertility decline: Islam, economics, and reproductive decision making among working-class Moroccan women. Med Anthropol Q. 2011;25(4):417-35.

26. Khalaf IA, Abu-Moghli F, Callister LC, Rasheed R. Jordanian women's experiences with the use of traditional family planning. Health Care Women Int. 2008;29(5):527-38.

27. Rustagi N, Taneja DK, Kaur R, Ingle GK. Factors affecting contraception among women in a minority community in Delhi: a qualitative study. Health Popul Perspect Issues. 2010;33(1):10-5.

28. Sapkota D, Adhikari SR, Bajracharya T, Sapkota VP. Designing evidence-based family planning programs for the marginalized community: an example of Muslim Community in Nepal. Front Public Health. 2016;4:122. 
29. Ying Zhang Erin E. McCoy· Roda Scego William Phillips· Emily Godfrey. A Qualitative Exploration of Somali Refugee Women's Experiences with Family Planning in the U.S. Journal of Immigrant and Minority Health (2020) 22:66-73.

30. World Health Organization (WHO), Programming Strategies for Postpartum Family Planning, Geneva: WHO, 2013.

31. Lockwood M. Structure and behavior in the social demography of Africa. Popul Dev Rev. 1995;21(1):1-32.

32. Egeh AA, Dugsieh O, Erlandsson K, Osman F. The views of Somali religious leaders on birth spacing A qualitative study. Sexual \& reproductive healthcare : official journal of the Swedish Association of Midwives. 2019;20:27-31.

33. Smith, H.J., Chen, J. \& Liu, X. Language and rigour in qualitative research: Problems and principles in analyzing data collected in Mandarin. BMC Med Res Methodol 8, 44 (2008). .

\section{Figures}
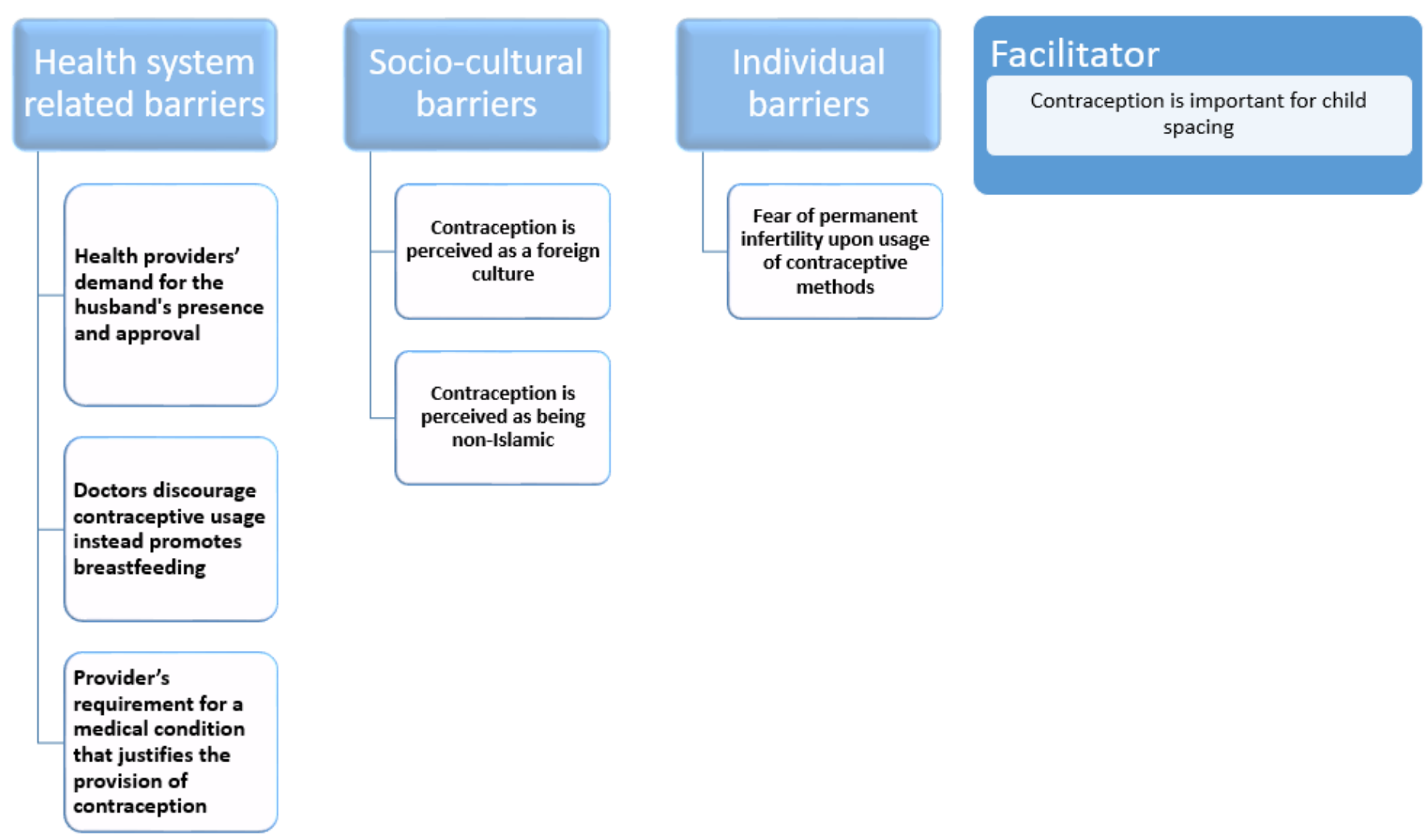

\section{Figure 1}

Barriers and facilitators to usage of contraceptive methods using ecological model 